\title{
SUR LES DONNÉES HISTORIQUES
}

La recherche historique n'a peut-être jamais revêtu autant d'intensité et de vigueur que de nos jours ; jamais non plus elle n'a prétendu embrasser d'aussi vastes secteurs du passé. Or, bien qu'elle s'empare des techniques les plus modernes pour explorer des territoires autrefois tout juste repérés, bien qu'elle accumule et diversifie sans cesse davantage les connaissances qu'elle engendre, elle se trouve soudain frappée d'impuissance dès qu'elle tente d'interpréter la masse d'informations qu'elle a collectée. Elle s'égare dans des débats sans fin sur la nature des événements ou sur les causes qui les provoquent. Elle se demande avec angoisse si cette société qu'elle étudie est "d'ordre " ou " de classes ", si l'origine de cette crise est "monétariste " ou " malthusienne ". Et quand, pour sortir de ces cercles infernaux, elle remet l'ouvrage sur le métier, elle fournit inlassablement des « preuves » qui, loin d'être décisives, satisfont également les tenants des thèses opposées. Est-ce faute de pouvoir dominer un trésor qui lui échappe à force de s'enrichir ? Ou ne serait-ce pas plutôt par manque de réflexion sur la matière de l'histoire et la manière de la maîtriser ? Car tout compte fait, l'historien s'interroge fort peu sur le contenu des données qu'il utilise et sur les concepts les plus propres à les manipuler. Toutefois, comme ceux-ci doivent s'adapter à celles-là pour prétendre à quelque efficacité, c'est donc par elles qu'il paraît logique de commencer et ce sont elles que cet essai désirerait aborder.

\section{DONNÉES HISTORIQUES ET ÉVÉNEMENTS}

Savoir en quoi consistent les données historiques suppose déjà qu'elles soient définies avec une netteté suffisante pour être reconnues comme telles sans contestations possibles. Leur appartenance à l'histoire lèverait toute hésitation si aucun doute ne planait sur l'objet de cette science. Or celui-ci subit malheureusement d'un auteur à l'autre assez de modifications pour que, sans entamer l'essentiel (le renvoi au 
passé), elles laissent néanmoins une marge non négligeable d'incertitude. Considérer l'histoire comme la connaissance des événements mémorables ou l'entendre comme $\mathrm{P}$. Veyne comme un « récit d'événements vrais " n'est pas exactement la même chose et la première proposition exige une sélection des données plus rigoureuse et d'un autre ordre que celle que réclame la seconde. Cependant si, au lieu de rapporter théoriquement les données historiques à l'histoire pour les définir, on les repère cette fois en fonction de la production historique existante, la difficulté disparaît d'elle-même puisqu'on renvoie à un corpus constitué, nettement déterminé à l'heure où l'on écrit, et non à une collection de possibles qui ne sont pas forcément encore réalisés et qui dépendent de la conception que chacun se fait et se fera de l'histoire. Aussi accepterons-nous ici pour données historiques, tout élément de connaissance sur le passé mis à jour par un historien ou quelqu'un agissant comme tel, utile à la compréhension de ce passé ou susceptible de le devenir.

Cette manière de procéder appelle néanmoins une remarque car elle sous-entend un choix qui pour être implicite n'en est pas moins réel. Elle ne préjuge pas, en effet, de la possibilité ou de l'impossibilité d'un savoir sur le passé ; elle ne se prononce également pas sur le bien-fondé de chaque donnée particulière avancée par tel ou tel historien. Cette attitude et les négligences qu'elle semble induire à première vue se justifient cependant pleinement par l'optique de cette étude. Il s'agit en effet ici d'étudier les éléments constitutifs d'un savoir et non pas les rapports que ces éléments entretiennent avec la réalité qu'ils sont censés représenter. D'ailleurs, il paraît difficile de poser la question de leurs rapports avec le passé sans déterminer leur propre contenu, car une erreur commise sur celui-ci risque de mettre en porte-à-faux tout examen critique de leurs relations avec la réalité. L'historien admet en général que ces éléments sont les " événements ". Mais cette " évidence », justement parce qu'elle est ressentie comme telle et acceptée ainsi sans contrôle, pourrait bien cacher, sinon une simplification abusive, au moins une imprécision source de confusions.

Certes, le trait fondamental et indiscuté des données historiques est qu'elles portent toutes sur des événements, c'est-à-dire sur des objets de connaissance du passé « individualisés » par « le fait qu'ils arrivent à un moment donné " ', autrement dit déterminés par une date. Dans cette perspective, l'ostracisme de Thémistocle survenu en $472 / 471$, la « démocratie athénienne » dans la seconde moitié du premier millénaire

1. Paul Veyne, Comment on écrit l'histoire. Essai d'épistémologie, Paris, Le Seuil, 1971 , p. 19. 
avant Jésus-Christ ou la " cité antique » pendant toute l'Antiquité classique, constituent tous, bien qu'à des titres divers, des événements puisqu'ils se situent à des époques précises nettement délimitées, quelle qu'en soit d'ailleurs la durée. Au contraire, la « démocratie » ne peut pas être considérée comme telle ; en effet, si elle existe, elle transcende les événements particuliers dans lesquels elle s'incarne. Cette transcendance, parce qu'elle est source d'intemporalité, l'exclut du champ d'observation historique.

\section{FAITS ET PHÉNOMĖNES}

Toutefois, les événements ne forment pas un ensemble parfaitement homogène. Suivant le niveau auquel on se place, deux catégories s'y distinguent nettement, que l'on peut baptiser d'un côté les " faits ", de l'autre les "phénomènes ». Les faits sont dans cette optique des événements bruts, c'est-à-dire tirés directement des documents quelle que soit la nature de ces derniers ; ainsi l'ostracisme de Thémistocle s'établit à partir de récits ou de tessons de poterie, l'assassinat de Henri IV, d'après des mémoires et des pièces officielles. Cette immédiateté du fait par rapport aux documents ne préjuge nullement de son authenticité, c'est-à-dire de ce qu'il soit effectivement survenu dans le passé à la date prétendue. Néanmoins, pour en réfuter la réalité, un historien doit également s'appuyer sur des documents, souvent d'autres quoique aussi parfois les mêmes, afin de prouver que le fait en question n'a pas eu lieu, ou au moins s'est déroulé selon des modalités différentes. Ainsi pour nier la concession au Xle siècle d'une charte urbaine, il faut par exemple découvrir que les formules utilisées dans cette charte n'ont pas été employées avant le XVe siècle, donc que le document n'a pu être rédigé au plus tôt qu'à l'extrême fin de la période médiévale. En bref, qu'un historien établisse un fait ou inversement en récuse la réalité, il procède toujours sans intermédiaire à partir des documents et d'eux seuls.

Il n'en est pas de même des phénomènes. Ceux-ci, en effet, dérivent de faits triés et regroupés en séries exploitées en tant que séries, au prix de ce que l'on pourrait nommer une "intégration sérielle de faits". Ainsi le phénomène " démocratie athénienne " se déduit du repérage des pratiques gouvernementales d'Athènes au ve siècle avant JésusChrist, de leur inclusion dans un ensemble qui les comprend toutes et elles seules, et de l'observation de cet ensemble en tant qu'ensemble et non pas comme une juxtaposition des pratiques considérées individuellement. De même, le phénomène "crises de mortalité à l'époque 
moderne " découle d'un recensement des augmentations violentes et anormales des sépultures auX XVI et XVII ${ }^{e}$ siècles, de leur rassemblement en un tout indépendant des lieux et des dates où elles se sont produites, et d'une étude de la collection ainsi constituée en tant que collection et non en tant que succession d'événements particuliers. Les techniques dites " sérielles » contemporaines n'opèrent pas autrement. Elles emploient seulement un instrument plus puissant, exigeant souvent une critique rigoureuse, les mathématiques. Par ailleurs, que les phénomènes dérivent des faits (et non plus comme ceux-ci immédiatement des documents) ne préjuge nullement de leur aptitude à représenter les événements du passé. Tout historien peut contester le bien-fondé de l'un d'entre eux ; toutefois, il ne le fait pas au nom des documents comme pour les faits, mais au nom des faits eux-mêmes ; il suffit qu'il en intègre de nouveaux qui changent les caractères de l'ensemble qui les regroupe, ou qu'il donne une autre analyse de la série qui les rassemble. Il a été ainsi possible de prétendre qu'il se rencontre au XVIIle siècle assez de rendements céréaliers stagnants pour refuser toute « Révolution agricole » en France à cette époque ${ }^{2}$. Néanmoins, que l'historien établisse un phénomène ou qu'il en récuse l'authenticité, il procède cette fois non plus directement d'après les documents, mais uniquement à partir des faits sériellement intégrés et, il faut ajouter, parfois même à partir d'autres phénomènes traités de façon identique.

En effet, la dérivation des événements les uns des autres ne s'exerce pas seulement des faits aux phénomènes ; elle s'accomplit également de phénomènes plus particuliers à un phénomène plus général. Les premiers se comportent alors fonctionnellement comme des faits bien qu'ils ne proviennent pas directement des documents. Il y a dans ce cas $«$ utilisation factuelle des phénomènes ". Ainsi opère Fustel de Coulanges dans sa Cité antique. Pour établir le sujet du premier chapitre du premier livre (les croyances sur l'âme et sur la mort), il se penche sur les rites de sépulture ; il dégage ceux-ci de textes littéraires dont les références occupent en moyenne, dans l'édition de 1883, une dizaine de lignes par page imprimée. Donc, d'une série de faits (à savoir une foule de pratiques individuelles constatées grâce à une multitude de témoignages qui sont autant de documents), il reconstitue un phénomène, les croyances sur l'âme et sur la mort. Jusqu'ici, rien de contradictoire avec l'affirmation que les faits découlent immédiatement des documents et que les phénomènes dérivent des faits par intégration sérielle de ceux-ci. Mais

2. Michel MORIneau, Les Faux-semblants d'un démarrage économique : agriculture et démographie en France au Xville siècle, Cahier des Annales, $\mathrm{n}^{\circ} 30$, Paris, Armand Colin, 1970, passim. 
Fustel de Coulanges ne s'en tient pas là car, confrontant à leur tour ces croyances sur l'âme et sur la mort avec d'autres phénomènes portant sur d'autres croyances et élaborés dans les mêmes conditions, il passe à un phénomène plus général, plus englobant, les " antiques croyances", sujet de son premier livre. Puis, ayant opéré d'une manière identique pour "la famille", il parvient à l'aide des phénomènes "antiques croyances " et "famille " à concevoir et à caractériser le phénomène " cité antique ". Celui-ci émane ainsi en dernier ressort d'une suite de dérivations successives de phénomènes à phénomènes, obtenues par analogies ou comparaisons.

\section{LES ÉVÉNEMENTS ET L'HISTORIEN}

En définitive, un phénomène n'est donc appréhendé qu'après au moins trois interventions de l'historien. Celui-ci, en effet, doit d'abord effectuer un choix entre des faits ou des phénomènes disponibles ; il lui faut ensuite les intégrer sériellement pour enfin en dégager un phénomène nouveau. Ainsi, ce dernier ne s'impose pas comme une évidence qu'un observateur passif se contenterait d'enregistrer ; il se place au contraire au terme d'un enchaînement d'opérations provoquées et conduites par l'historien lui-même. En l'élaborant à partir d'éléments préalablement triés et regroupés, celui-ci s'en affirme de fait pleinement l'inventeur. Aussi un phénomène n'est-il en aucune manière une donnée immédiate, partant incontestable, mais une construction grâce à laquelle on espère rendre plus compréhensible un fragment du passé.

Fondamentalement, quoique au terme d'une procédure différente, il n'en va pas autrement des faits. En dépit des apparences, eux non plus n'ont rien d'évidences qu'il suffirait de constater dans les documents. Pour voir le jour, ils exigent également la participation active et volontaire de l'historien. S'il n'en était pas ainsi, les contestations, que l'un d'entre eux suscite de temps à autre, resteraient entièrement incompréhensibles. Surtout, d'un même document, les historiens ne tirent que progressivement, au cours des lectures des générations successives, les faits qui s'y cachent. Ils n'y retiennent que ceux qui leur conviennent et ignorent les autres. Ainsi, dans les registres paroissiaux, à travers le recensement des baptêmes, des bénédictions nuptiales et des sépultures, certains n'ont d'abord vu que des naissances, des mariages et des décès. D'autres, en revanche, y aperçurent par le biais des signatures des hommes plus ou moins alphabétisés. D'autres encore y repèrent maintenant, en considérant les témoins, les parrains ou les dévolutions de prénoms, des relations intra- et interfamiliales. D'autres, enfin, y trouvent des 
préférences sociales en notant les milieux respectifs des conjoints. Les documents contiennent donc en puissance une multitude de faits ; néanmoins ceux-ci ne voient le jour que quand l'historien s'intéresse à eux. Les faits ne constituent pas plus une donnée immédiate que les phénomènes. Il n'y a d'événements que ceux découverts par l'historien. Les autres demeurent potentiels ; ils forment un « champ non événementiel » infini que grignote le « champ événementiel » sans jamais parvenir cependant à le réduire totalement ${ }^{3}$.

\section{LE RÔLE DU PROJET DE L'HISTORIEN}

Les événements ne s'imposent pas d'eux-mêmes. Que l'historien soit obligé d'intervenir pour les repérer, les place sous la dépendance de ce qui provoque leur découverte, c'est-à-dire de ce qui conduit le chercheur à les distinguer dans ce qui est arrivé, à les isoler et à les relever, alors qu'il ignore et oublie tous les autres. Or, ce qui guide l'historien dans cette sélection, c'est son projet hic et nunc, c'est-à-dire ce qu'il décide à un moment d'étudier du passé. Par exemple, celui qui cherche à reconstituer le mouvement de la production frumentaire avant les grandes statistiques générales du XIXe siècle tente de s'appuyer soit sur les variations des rendements, soit sur les fluctuations du prẻlèvement décimal. S'il opte pour la première solution, il perçoit comme utiles et relève les rendements à la semence. S'il se tourne au contraire vers la seconde, il les délaisse au bénéfice des produits de dîmes en nature.

Cette activité sélective se rencontre sans équivoque possible dans l' " histoire-problème ", dans l' " analytical History ". Dans ce cas, en effet, l'historien pose lui-même une question qu'il s'efforce ensuite de résoudre. Il prélève alors dans ce qui est arrivé ce qui lui paraît utile pour atteindre son but et n'en retient que ce qu'il juge pertinent. Cependant, à chaque avancée de son travail et en fonction des résultats obtenus, il peut changer le questionnaire. Aussi le projet réside-t-il moins à une étape donnée dans la problématique initiale que dans les hypothèses de travail avouées ou inavouées qu'il éprouve successivement. Ce sont elles et elles seules qui l'autorisent à reconnaître tel ou tel événement comme convenable à son propos. Ainsi, se demander ce qui provoque la hausse tendancielle des prix européens au $X \mathrm{XI}^{e}$ siècle conduit l'historien à observer des événements différents selon les hypothèses qu'il formule et essaie ensuite de tester. L'hypothèse quantitativiste le pousse à retenir

3. P. VEYNE, op. cit. supra n. 1, passim. 
les flux de métaux précieux et plus spécialement les arrivages américains ; l'hypothèse malthusienne en revanche le porte vers l'évolution de la population, la courbe des disponibilités alimentaires et la répartition sociale de ces dernières. En conséquence, dans l'histoire-problème, ce qui influe immédiatement sur le repérage et la sélection des événements, ce n'est pas tant le projet initial (la problématique de départ) que les projets dérivés successifs (les hypothèses de travail), le premier n'agissant qu'en suscitant les seconds.

Il en va de même pour les autres formes d'histoire, y compris celle qui, aux yeux des Anglais, constitue l'antithèse de l'analytical History, cette narrative History qui se proclame uniquement descriptive, simple exposé et pur rappel des événements du passé. En effet, en dépit de ses affirmations et de son "intime conviction ", l'historien qui s'y livre opère en réalité un nombre considérable de sélections, rarement explicites et trop fréquemment inconscientes, toutes en étroits rapports avec le projet qu'il s'est en fait lui-même subrepticement fixé. Une première série de sélections découle du sujet qu'il a adopté ; celui-ci impose un découpage dans le temps et dans l'espace et le choix d'un genre d'histoire. Celui qui veut de cette manière traiter l'histoire politique du Second Empire isole la France et la période s'écoulant du 2 décembre 1852 au 4 septembre 1870. De plus, il ne conserve que les événements " politiques " ou plus exactement ceux qu'il estime tels car il a vite à faire face à nombre de cas douteux si bien qu'il ne sait pas toujours lesquels il doit garder ou au contraire éliminer.

Une seconde série de sélections, plus subtile, moins reconnue, peutêtre capitale, intervient quand notre adepte de la narrative History entreprend justement son récit. Il lui faut, en effet, négliger tel fait et privilégier tel autre, articuler sa narration autour de quelques pivots (les événements-clés) auxquels il accorde une importance particulière, révélant ainsi une interprétation souvent inavouée. Il lui serait d'ailleurs impossible de procéder différemment ; s'il s'y essayait, il disparaîtrait vite sous une avalanche événementielle comme l'érudit de "L'île aux pingouins » sous les cataractes de fiches qu'il avait accumulées. En bref, même s'il le désire, même s'il y croit, il ne peut pas néanmoins tout raconter, il doit se contenter de ceci et refuser cela, ce qui l'entraîne à choisir ceci et à abandonner cela dans ce que contiennent les sources qu'il consulte. Là encore, c'est son projet, un projet cette fois exceptionnellement exprimé, la plupart du temps même pas entrevu qui, comme pour l'histoire-problème, commande sa démarche, ne lui faisant prendre dans ce qui est autrefois survenu que ce qu'il juge convenable pour son propos.

De cette nécessité, Barante en son temps, dans la préface de son His- 
toire des Ducs de Bourgogne, donne un exemple curieux, involontaire si on prend au pied de la lettre ses explications, conscient si l'on admet qu'il use du double langage alors habituel aux libéraux. Il commence par évoquer les « histoires où le génie philosophique suit à travers tous les faits successifs le développement d'une idée ou le progrès d'une cause " et qui tirent ainsi « leur beauté " "surtout » de "l'unité de conception ", de " cette puissance de l'auteur qui distingue et ordonne les faits selon sa pensée, selon le but de ses recherches et de son analyse " ${ }^{4}$. Puis il remarque qu'il vit « dans un temps de doute » où " les opinions absolues sont ébranlées », où " ce ne sont plus des jugements et des opinions qu'on semble attendre de celui qui veut essayer cette tâche (écrire l'histoire) " 5 , où « ce que l'on veut d'elle (l'histoire), ce sont des faits " ${ }^{6}$. Il décide, en conséquence, (ou feint de décider) de "présenter une peinture fidèle d'un des siècles de notre histoire " , " une narration suivie, complète, exacte " ${ }^{8}$. Cependant, il ajoute quelques lignes plus bas qu'il n'a « rien souhaité autant que de représenter l'opinion publique, ses vicissitudes, ses progrès, son influence » puisque de cette manière son étude « fait entrer dans l'histoire le mobile le plus puissant et, si l'on peut ainsi parler, son principal personnage ", car « même dans ces temps barbares où régnait la force, où l'inégalité entre les droits que les hommes ont à la justice, était une croyance admise par tous; dans ces temps où les communications entre les citoyens d'une même patrie étaient si imparfaites, la pensée et la voix du peuple exerçaient déjà un immense pouvoir " ${ }^{9}$. En toutes circonstances, le projet de l'historien le conduit donc à remarquer certains événements et à passer à côté d'autres sans même les apercevoir.

\section{LA CONNAISSANCE HISTORIQUE DES ÉVÉNEMENTS}

La procédure d'invention des événements telle qu'elle vient d'être dégagée et décrite détermine, en outre, la connaissance qu'en obtient l'historien. Les relevant non pas à l'occasion de son projet, mais uniquement en fonction de celui-ci, il ne les repère pas pour eux-mêmes, mais exclusivement pour les renseignements qu'ils lui procurent. Autrement

4. M. DE Barante, Histoire des ducs de Bourgogne de la maison de Valois, 1364-1477, Paris, de Normant et Garnier frères, $7^{\mathrm{e}}$ éd., 1854, t. 1, p. 10-11.

5. Ibid., p. 33.

6. Ibid., p. 35 .

7. Ibid., p. 38 .

8. Ibid., p. 39 .

9. Ibid., p. 40-41. 
dit, s'il ne les découvre que quand il en a besoin, il ne les découvre aussi que dans l'exacte mesure où il en a besoin. Il acquiert donc, en réalité, non pas un corpus d'événements mais une collection d'informations sur des événements. Lorsque l'école française de démographie historique a pris comme hypothèse de travail que les populations du royaume parvenaient à contrôler leur fécondité aux $\mathrm{XVII}^{e}$ et $\mathrm{XVIII}^{e}$ siècles, elle a découvert l'âge tardif des femmes à leurs premières noces et le faible niveau des conceptions hors mariage. De ces deux événements ainsi mis au jour, elle ne retient cependant que leur rôle de freins à la croissance démographique, c'est-à-dire l'information qui lui paraît utile pour sa problématique. Que ces événements aient ou non provoqué des refoulements, favorisé la masturbation ou développé l'agressivité la laissait indifférente. Cette toute-puissance du projet sur la connaissance que l'on a des événements se marque jusque dans le lapsus révélateur. Ces mêmes historiens démographes baptisent " naissances illégitimes » les seuls accouchements des filles célibataires. En effet, en raison même de leur projet, ne devient pour eux " illégitime » que tout ce qui menace l'influence régulatrice sur le niveau de la population de l'âge tardif au mariage. Qu'importe que l'enfant soit l'œuvre de l'époux ou de l'amant, si la femme ne procrée que dûment mariée à une date déjà avancée de sa vie. En conclusion, le projet de l'historien guide celui-ci vers tel événement et non tel autre, mais il le conduit également à ne s'y intéresser que pour les renseignements qu'il peut y trouver. Il le contraint ainsi à une double sélection, d'une part au niveau de ce qui est arrivé dans le passé, d'autre part au niveau des informations que peut lui fournir ce qu'il a retenu.

Ainsi, l'achèvement de chaque projet fournit sur tout événement qu'il reconnaît pour sien, une ou des informations et uniquement cette ou ces informations. Cependant, en fonction de sa propre recherche et des travaux antérieurs un historien parvient à posséder sur un événement un faisceau de renseignements plus ou moins vaste qui en révèle des aspects plus ou moins nombreux et plus ou moins variés, progressivement dégagés au fil des études successives qui l'utilisèrent. Considérons par exemple la hausse séculaire des prix au $X v 1^{e}$ siècle. L'historien sait désormais qu'elle fut tendancielle, coupée d'intercycles, d'une croissance discontinue, qu'elle affecta plus les denrées alimentaires et parmi celles-ci les plus courantes que les produits industriels et surtout les salaires journaliers ou à la tâche, qu'elle se situa lors d'arrivages massifs de métaux précieux de Guinée d'abord, d'Amérique ensuite, mais aussi quand les disponibilités et la productivité diminuèrent sur long terme, qu'elle autorisa des « bénéfices d'inflation » pour nombre de marchands quoique en revanche, elle fit baisser le pouvoir d'achat des rentes fixes prescrites en numéraire ; et l'historien connaît sur elle bien d'autres choses 
encore, vu que cette hausse se place à un carrefour où se croisent de multiples entreprises historiques. En dépit de cette abondance, de nouveaux projets une fois exécutés livreront d'autres renseignements, et comme le nombre de ces projets est imprévisible et inépuisable, le nombre de renseignements possibles sur cette hausse des prix (mais ce serait également vrai de tout autre événement) est lui aussi imprévisible et inépuisable. En effet, l'expérience historiographique passée montre que le « questionnaire " le plus mûrement réfléchi et le plus rigoureusement établi n'atteint jamais l'exhaustivité absolue, car il est bien incapable de prévoir les chemins sur lesquels s'aventurera la recherche postérieure, donc les problèmes qu'elle sera conduite à poser et qu'elle tentera ensuite de résoudre. C'est pourquoi, quand ce qui est arrivé dans le passé devient un événement, c'est-à-dire quand il apparaît dans le champ historique, les informations qu'il donne sont en procès d'expansion indéfinie quel qu'en soit le rythme.

En conclusion, comme l'historien ne recueille que des informations sur les événements qu'il repère, il en résulte qu'il ne connaît jamais ceuxci en eux-mêmes, que pour lui ils « ne sont pas des choses, des objets consistants, des substances ", qu' " ils n'existent... pas avec la consistance d'une guitare ou d'une soupière " ${ }^{10}$. Autrement dit, les données historiques, tout en portant sur des événements, ne peuvent en aucune façon coïncider avec ces objets insaisissables. Elles doivent se borner à des informations sur leur compte, ce qui revient à dire qu'elles ne les contiennent jamais intégralement.

\section{LES MISES EN RELATION}

D'un autre côté, si les informations sur un événement correspondent à ce que l'historien en connaît, elles ne constituent cependant pas elles aussi des entités qui se suffiraient à elles-mêmes. Au contraire, elles doivent leur seule existence aux relations que les historiens ont établies entre cet événement et d'autres événements en exécutant leurs projets successifs. En d'autres termes, elles ne forment pas des objets autonomes, donc disponibles tels quels, sans autre référence qu'à l'événement considéré. En réalité, chacune d'elles exprime une liaison nouée entre cet événement et au moins un autre événement, et c'est cette liaison, et cette liaison seule, qui lui donne un sens, mieux qui lui permet de voir le jour. La raison en est simple : les informations sur les événements naissent de la mise en œuvre des projets historiques. Or un projet historique

10. P. VEYNE, op. cit. supra n. 1, p. 52. 
se définit toujours comme une recherche de rapports existants entre des événements, car ce sont ces rapports une fois établis qui autorisent un récit, permettent de résoudre un problème ou de comprendre un phénomène.

Fustel de Coulanges l'écrit clairement dans l'introducton particulièrement lucide qui ouvre la Cité antique :

" Regardez les institutions des anciens sans penser à leurs croyances, vous les trouverez obscures, bizarres, inexplicables [...] Mais en regard de ces institutions et de ces lois, placez les croyances ; les faits deviennent aussitôt plus clairs, et leur explication se présentera d'elle-même. [...] La comparaison des croyances et des lois montre qu'une religion primitive a constitué la famille grecque et romaine [...] Cette même religion, après avoir élargi et étendu la famille, a formé une association plus grande, la cité, et a régné en elle comme dans la famille $" ~ " 1 !$.

Ce thème se retrouve à la fin de l'œuvre quand Fustel de Coulanges conclut du triomphe du christianisme que

« par cela seul que la famille n'avait plus de religion domestique, sa constitution et son droit furent transformés; de même que par cela seul que l'État n'avait plus sa religion officielle, les règles du gouvernement des hommes furent changées pour toujours $\gg 12$.

Dans ces conditions, ce que nous lègue l'auteur sous le vocable de "Cité antique ", c'est un système de relations qui aurait existé dans le monde gréco-latin entre les croyances, la famille et la cité. Que ce système constitue ou non l'essence réelle ou supposée du phénomène "Cité antique ", qu'il l'épuise ou ne l'épuise pas, importe certes beaucoup à la connaissance historique, mais fort peu du point de vue qui est ici le nôtre. En revanche, ce que nous devons retenir, c'est que dans ce système, les croyances, la famille et la cité ne sont saisies non seulement qu'en fonction les unes des autres mais, en fait, plus restrictivement encore, que dans un rapport singulier nettement défini ayant pour base une « religion primitive » qui " constitue » la famille et indirectement par élargissement de celle-ci, la cité. Il s'ensuit que les renseignements que fournit Fustel de Coulanges sur ces croyances, cette famille et cette cité ne sont compréhensibles et interprétables qu'en tenant compte du système qu'il a élaboré, c'est-à-dire des connexions qu'il a établies entre

11. Numa Fustel de Coulanges, La Cité antique, Paris, Hachette, rééd. 1969, p. 3-4. 12. Ibid., p. 464. 
elles, tout simplement parce qu'il ne conserve et même ne voit de chacune d'elles que ce qui lui sert à la mettre en relation avec les autres.

La démarche qu'adopte Fustel de Coulanges dans sa Cité antique se montre particulièrement consciente et explicite. D'autres le sont tout autant, spécialement dans l'historiographie contemporaine. Cependant, même dans cette dernière, beaucoup restent si implicites, si inconscientes, tellement cachées qu'il n'apparaît pas toujours évident que la finalité de tout projet historique consiste ipso facto à établir des relations entre des événements, que le praticien le veuille ou non. Pour le prouver irréfutablement, il faudrait bien entendu passer au crible l'ensemble de la production historique, tâche manifestement impossible, car proprement surhumaine au sens le plus strict. Évidemment, certains cas ne laissent aucun doute. Ainsi tous ceux qui insistent depuis Jean Bodin et son "Paradoxe de Monsieur de Malestroit » sur le synchronisme entre les arrivages américains de métaux précieux et la hausse tendancielle des prix européens au $X \mathrm{XI}^{\mathrm{e}}$ siècle, visent essentiellement à fonder un rapport de cause à effet entre ces deux événements. Mais il n'en est pas toujours de même. Aussi, ne pouvant envisager la totalité des occurrences douteuses, il a paru préférable de se concentrer ici sur celle qui semble la plus discutable, à savoir la " narrative History " la plus rigide, la plus intransigeante, qui prétend ne présenter qu'une pure et simple description d'événements successifs.

Or, écrire une narration historique revient également à mettre en relation des événements entre eux. Le seul fait de raconter équivaut à établir une succession d'événements, donc à saisir ceux-ci dans des rapports chronologiques d'antériorité ou de simultanéité. D'ailleurs, beaucoup d'auteurs doublent ces rapports d'un autre rapport, de causalité cette fois. Car craignant à juste titre le ridicule, la plupart des auteurs ne se contentent pas, par exemple, d'enregistrer qu'une colère de Louis XIV suit une requête du duc de Lauzun. Ils écrivent tout bonnement que cette requête a entraîné, a provoqué la fureur royale, introduisant ainsi, plus ou moins subrepticement, un enchaînement de cause à effet. En conséquence, ceux qui prétendent se limiter à un récit uniquement descriptif, posent, eux aussi, des rapports entre les événements et ne saisissent ceux-ci que par et à travers ces rapports.

En définitive, ce que livre tout travail historique achevé, ce sont essentiellement des rapports entre des événements, des événements qui sont selon la forte expression de P. Veyne des « nœuds de relations ». Partant, ces rapports constituent les éléments fondamentaux de la connaissance historique qui, sans eux, n'existerait pas. Il en résulte que si on entend par donnée historique «tout élément de connaissance sur le passé mis à jour par un historien et utile à la compréhension de ce 
passé ", il faut bien admettre alors que ces rapports coïncident avec les données historiques. Celles-ci sont donc tout à la fois plus que des événements (puisqu'elles sont essentiellement des relations entre des événements) sans pour autant les contenir intégralement (car elles ne les considèrent qu'en tant que " nouds de relations »).

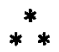

S'il en est ainsi, l'historien à force d'établir des relations entre les événements finit par les prendre dans un réseau complexe qui constitue le vrai savoir historique. Il y parvient selon deux modalités différentes. D'un côté, il peut s'appuyer sur un événement privilégié qui sert de pivot à son travail ; ainsi a-t-on tissé autour de la hausse des prix du $\mathrm{XVI}^{\mathrm{e}}$ siècle une toile qui enserre en même temps la production et le commerce des métaux précieux, leur thésaurisation, la croissance démographique et le recul de la productivité agricole. D'un autre côté, il lui arrive également de créer des chaînes dont les maillons sont des événements. Des générations successives de chercheurs ont de cette manière raccordé pour les $\mathrm{XVII}^{e}$ et $\mathrm{XVIII^{e }}$ siècles français, crise frumentaire et crise démographique, crise démographique et régulation du volume de la population, régulation de ce volume et fécondité des femmes, fécondité et âge tardif au premier mariage et faiblesse des conceptions prénuptiales. À chaque fois, quel que soit le procédé utilisé, l'historien élabore ainsi peu à peu au prix de retouches plus ou moins profondes et de liaisons nouvellement découvertes, des systèmes relationnels toujours modifiables et sans cesse en expansion. Le savoir historique est donc à un moment donné, l'état de connaissance à ce moment donné du champ relationnel unissant les événements historiques. Sa maîtrise suppose en conséquence la recherche de concepts capables de rendre compte de ce champ et de le dominer et non pas de rendre compte et de dominer les événements, ni même telle ou telle relation particulière isolée des autres. Il n'est pas sûr que les historiens possèdent à l'heure actuelle le matériel suffisant pour y parvenir. Il paraissait cependant nécessaire, avant même de songer aux outils, de déterminer les caractères des objets auxquels ils seraient appliqués. C'est ce que cet essai a voulu tenter ; qu'il y soit parvenu, c'est ce que le lecteur jugera.

Hugues NEVEUX, Université de Paris $X$-Nanterre. 